\title{
Adaptive Multiscale Ultrasound Compounding Using Phase Information
}

\author{
Vicente Grau and J. Alison Noble \\ Wolfson Medical Vision Laboratory, Department of Engineering Science, \\ University of Oxford, Parks Road, Oxford OX1 3PJ, \\ \{vicente, noble\} arobots.ox.ac.uk
}

\begin{abstract}
The recent availability of real-time three-dimensional echocardiography offers a convenient, low-cost alternative for detection and diagnosis of heart pathologies. However, a complete description of the heart can be obtained only by combining the information provided by different acoustic windows. We present a new method for compounding 3D ultrasound scans acquired from different views. The method uses multiscale information about local structure definition and orientation to weight the contributions of the images. We propose to use image phase to obtain these image characteristics while keeping invariance to image contrast. The monogenic signal provides a convenient, integrated approach for this purpose. We have evaluated our algorithm on synthetic images and heart scans from volunteers, showing it provides a significant improvement in image quality when compared to traditional compounding methods.
\end{abstract}

\section{Introduction}

Real-Time 3D Echocardiography. Several imaging modalities (X-ray, angiography, MRI, 2D echocardiography) have been proven to be useful in cardiology. However, echocardiography presents unique characteristics that make it the most commonly applied imaging diagnostic technique in clinical practice. Traditionally, twodimensional echocardiography has been used widely as a relatively cheap, portable and real-time interactive assessment of heart function. Using a 2D imaging modality introduces disadvantages such as the difficulty to characterize three-dimensional structures or the dependence on the probe position, which makes it difficult to compare images if acquired by different clinicians or at different times.

Recently developed technology has allowed, for the first time, to obtain threedimensional images of the heart in real time. This new imaging modality opens a wide range of possibilities for echocardiography in clinical routine. However, at its present stage, due to the limited field of view of the transducer, it is not possible to scan the whole heart in a single acquisition (and, in some cases, not even the left ventricle). Furthermore, some cardiac structures can be appreciated only from particular acoustic windows. For this reason, a complete diagnostic study in real time 3D ultrasound (RT3DUS) will consist of several acquisitions obtained from different positions. The development of tools to combine these acquisitions and present a single, optimal dataset to the clinician could greatly improve the clinical uses of the technology. 
Compounding Ultrasound Scans. Compounding ultrasound images acquired at different times or from different locations is a common way to improve image quality, mainly through speckle reduction. Though using directly RF data for compounding is also possible, these data are not usually available in the clinical environment, so our research focuses on compounding intensity images. Usually, simple techniques, such as the mean or the maximum intensity, are used to combine the images. Little work has been done to explore alternative, more sophisticated compounding techniques. In [1], the authors propose to exploit the dependence of echo intensity on the angle of incidence of the ultrasound beam on a reflecting surface. Resulting images were significantly enhanced when compared to using the mean value to combine images. However, that method is based on an initial fitting of a surface to the data. This makes it difficult to apply to complex images, such as the cardiac ones, in which several structures are present and segmentation is a challenge.

Phase-Based Image Analysis Using the Monogenic Signal. Phase-based analysis has been proposed as an alternative to intensity for many image processing tasks [4]. Phase provides invariance to changes in brightness and contrast within the image: this property makes it particularly fit for ultrasound images, in which beam attenuation is present and echo intensity depends on the angle of incidence of the ultrasound beam. Local phase is usually calculated by combining the output of a set of filters with different angles. In [2], the authors introduce a new approach to calculate phase in ndimensional signals: the monogenic signal, an isotropic extension of the analytic signal which preserves its basic properties. Analogous to the analytic signal for 1-D, to build the monogenic signal the Riesz transform, a generalization of the Hilbert transform for higher dimensional signals, is used. In the frequency domain, the Riesz transform of an $\mathrm{N}$-dimensional image can be calculated by multiplying the original image with the filters $H_{i}$ :

$$
H_{i}(\boldsymbol{u})=\frac{u_{i}}{|\boldsymbol{u}|},
$$

where $\mathbf{u}=\left[\mathrm{u}_{1} \ldots \mathrm{u}_{\mathrm{N}}\right]^{\mathrm{T}}$, with $\mathrm{u}_{\mathrm{i}}$ representing the ith coordinate unit vector; there are thus as many filters as image dimensions. The monogenic signal is the combination of the original signal and the Riesz transform, so it assigns an N+1-dimensional vector to each spatial location. The angles defined by this vector represent the local phase and structure orientation, respectively. A complete explanation of the mathematical foundations and the equations needed to calculate phase and orientation from the monogenic signal can be found in [2].

In practice, it is interesting to localize features both in space and in frequency. To achieve this purpose, band-pass filters $G_{s}(u)$ are combined with the $H_{i}(u)$ filter in (1). A comprehensive analysis of different alternatives for band-pass filters was done in [3].

In this paper, we propose a novel compounding algorithm for ultrasound images in general, and for multi-view, RT3DUS echocardiography images in particular. The aim of the compounding algorithm is to maximize the information content of the combined images, for which we preserve those images which higher feature saliency; furthermore, we assign weights depending on the incidence angle of the ultrasound beam. We explore the use of phase to obtain information about both feature saliency and orientation: the monogenic signal constitutes a convenient 
framework for this purpose. Preliminary results on both phantom and real-time 3D cardiac images are presented, showing significant improvement over common compounding techniques.

\section{Compounding Using Feature Saliency and Orientation}

Image Registration. Registering echocardiographic images obtained from different windows is a non-trivial task. Both temporal and spatial registration are required. In this paper, however, we focus on the combination of images, so we assume they have been previously registered with enough accuracy. For the algorithm evaluation presented in Section 3, we performed an initial rigid registration by aligning manually selected landmarks (the valve rings) followed by a manual fine correction of the result. For the temporal registration, we identified corresponding frames in the sequences, and performed a linear interpolation between them.

Compounding Algorithm. In order to maximise the information content of the compound image, we need to keep the features which appear only in a subset of the original $\mathrm{N}$ images, without degrading these features by averaging them with the rest. Furthermore, it is well known that the backscattered energy, and thus the ultrasound image appearance, depends on the incidence angle (the angle formed by the ultrasound beam and the normal to the surface at the incidence point). Averaging the intensities obtained from two different views is not an optimal solution, as it would degrade the strong echoes generated by small incidence angles by introducing weaker echoes from more oblique incidences. Thus, where several images contain significant features, we should combine them giving preference to the ones in which the normal to the detected feature is better aligned with the ultrasound beam. For this purpose, we define two measures, $S_{i}(r)$ and $M_{i}(r), 0 \leq S_{i}(r) \leq 1,0 \leq M_{i}(r) \leq 1$, which quantify how important the information contained by image $i$ at position $r$ is, and how well aligned features at this location are with the ultrasound beam, respectively. The way we calculate $S_{i}$ and $M_{i}$ in this study is described below. We can then define the following rules to identify the characteristics of an image point, and thus select the best strategy for combining the values:

- If $S_{i}$ values are low in all images but one, the value from this image is adopted.

- If $S_{i}$ values are high in more than one image, the values in the high $S_{i}$ images are combined according to their respective alignment values $M_{i}$ (those images in which the point is better aligned will contribute a higher amount to the combination).

- If $S_{i}$ values in all images are low, the point is treated as pure speckle, so the average value is taken.

Here, the $S_{i}$ value is treated as the primary condition, and only in the case that it is not sufficient to determine the intensity value by itself, the alignment values $M_{i}$ are considered. We can transform the if-then conditions described above into equations to calculate the weight that each image should have in the final compound image, if we treat $S_{i}$ and $M_{i}$ as probabilities of having a significant feature and having a well- 
aligned feature, respectively. This can be done in a multiscale way by calculating multiscale decompositions of the images, applying the compounding process individually to each scale, and finally recombining the compound multiscale images. In the following, for simplicity, we have omitted scale subindices. As an example, we show the weighting factor for image $1, \lambda_{1}$, in the case that three images are combined:

$$
\begin{gathered}
\lambda_{1}=\left[S_{1} \bar{S}_{2} \bar{S}_{3}+S_{1} S_{2} \bar{S}_{3} M_{1} \bar{M}_{2}+S_{1} \bar{S}_{2} S_{3} M_{1} \bar{M}_{3}+S_{1} S_{2} S_{3} M_{1} \bar{M}_{2} \bar{M}_{3}\right]+\cdots \\
\cdots+\frac{1}{2}\left[S_{1} S_{2} \bar{S}_{3} M_{1} M_{2}+S_{1} \bar{S}_{2} S_{3} M_{1} M_{3}+S_{1} S_{2} S_{3} M_{1}\left(M_{2} \bar{M}_{3}+\bar{M}_{2} M_{3}\right)\right]+\cdots \\
\cdots+\frac{1}{3}\left[S_{1} S_{2} S_{3}\left(M_{1} M_{2} M_{3}+\bar{M}_{1} \bar{M}_{2} \bar{M}_{3}\right)\right]+\frac{\alpha}{3}\left[\bar{S}_{1} \bar{S}_{2} \bar{S}_{3}\right]
\end{gathered}
$$

with $\bar{S}_{i}=\left(1-S_{i}\right)$. Note that $S_{i}, M_{i}$ are not Boolean variables, but continuous probability values.

We can intuitively interpret the four main terms in (2). The first three terms in square brackets correspond, respectively, to the probabilities of having just one, two or three of the images (always including image 1) containing significant, well-aligned features. Finally, the last term represents the probability of having no structural information (i.e., pure speckle) at this point. We have introduced here a coefficient $0 \leq \alpha \leq 1$ which can be used for noise reduction. Note that $\alpha=1$ corresponds to an averaging of all images (the same effect produced by average compounding, but in this case applied only to regions with no feature information), while $\alpha=0$ produces a total elimination of detected speckle in the compound image. It is easy to generalize the above equation for any number of images. In our algorithm, a coefficient $\lambda_{i s}$ would be calculated for each image $i$ and for each scale $s$, using alignment coefficients $M_{i s}$. Finally, the compound image is calculated by combining all scales.

Using phase to calculate the feature significance and alignment parameters. The evolution of a phase measure across spatial scales can be used as a clue to differentiate image features from noise. One of the possibilities that have been proposed for this purpose is phase congruency [4].

$$
P C(\bar{r})=\max _{\bar{\varphi}(\bar{r}) \in[0,2 \pi]} \frac{\sum_{s} A_{s}(\bar{r}) \cos \left(\varphi_{s}(\bar{r})-\bar{\varphi}(\bar{r})\right)}{\sum_{s} A_{s}(\bar{r})}
$$

where $A_{s}$ represents the amplitude of the s-th Fourier component of the signal, and $\varphi_{s}$ is the local phase of the Fourier component at position $\bar{r}$. This parameter quantifies phase change over different scales. In [5], an alternative way to calculate the phase congruency was given. Phase congruency quantifies how phase varies across scales: a high value corresponds to a consistent phase value and is thus an indicator of a strong image feature. The utility of phase congruency to detect edges and lines in ultrasound images has been demonstrated before [6]. If the adequate scales are selected, phase congruency can be used to detect larger-scale structures, such as the ventricular walls in echocardiographic images. We can thus use $S_{i}(\bar{r})=g\left(P C_{i}(\bar{r})\right)$ in Equation 2, where $g($.) can be any monotonic function with $g(0)=0$ and $g(1)=1$. 
As described in Section 1, the monogenic signal can be also used to calculate the orientation of image features. The incidence angle at scale $s$ is defined as the angle between the ultrasound beam and the local image orientation at scale $s, \theta_{s}$. We define the parameter $M_{i s}=\cos \left(\theta_{i s}-\varphi\right)$, where $\varphi$ is the angle of the ultrasound beam.

To construct the multiscale image description required, many alternatives for bandpass filter selection exist [3]. We have chosen the Difference of Gaussians (DoG), which allows us to easily recombine the band-pass images. The filter bank is formed by S filters: S-2 band-pass filters, plus a low-pass filter and a high-pass filter which are used to generate a complete representation of the original image. All band-pass filters in the bank are constructed with an equal bandwidth value. In the experiments presented, we have found $\mathrm{S}=5$ to be an adequate value.

\section{Results and Discussion}

Tests Using a Synthetic Phantom. Simulated images were generated using the Field II program [7]. An elliptical ring phantom was generated and scanned using a simulated $5 \mathrm{MHz}$ sector probe. In Fig. 1, the results of compounding two images, acquired with a difference of $80^{\circ}$, are shown. Improvement of contrast and better edge definition, when compared to intensity averaging, can be clearly appreciated. We calculated contrast to noise ratio (CNR) as the difference between the mean values of the ring and the background, divided by the standard deviation of background intensity. CNR obtained with our method is $37 \%$ higher than with intensity averaging. An important indicator of the quality of our method is its effect on the magnitude and direction of the intensity gradient at the object contours: this is a crucial parameter for edge-based segmentation methods. We have calculated the intensity magnitude gradient in the direction normal to the ring contour. The increase in this parameter in our method, compared to intensity averaging, is shown in Fig 1(h): where the differences in alignment are high, increases of more than $30 \%$ are obtained.

Tests on Three-Dimensional Real-Time Echocardiographic Images. Finally, we applied our algorithm to RT3DUS images of the heart. Fourteen datasets were obtained by scanning two volunteers from both the apical and the parasternal windows. Images were registered and combined as explained in Section 2. Results from one of the volunteers are shown in Fig 2. When compared to intensity averaging (Fig 2(b)), our method with $\alpha=1$ (Fig 2(e)) shows a superior definition of significant heart structures. We have used smaller values of $\alpha$ to test the speckle reduction behaviour of the algorithm in a qualitative way: in Fig 2(c), where $\alpha=0.9$, speckle is reduced without a decrease in contrast in the important features. Finally, with $\alpha=0.6$ (Fig 2(f)), the most salient features are predominantly kept. The reader can better appreciate the improvement in the time sequences available online at http://www.robots.ox.ac.uk/ mvl/htdocs/research/gallery.html.

Discussion. We have presented a method for compounding ultrasound images acquired from different views. The novel aspect of our work is that, unlike the traditional methods used for compounding (average, maximum,...) we introduce information about the structural content of the image and its orientation. We also propose a particular way to calculate this information, based on the image phase, and implement it in an 


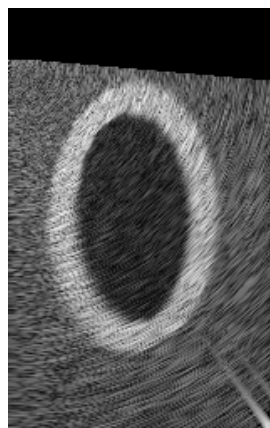

(a)

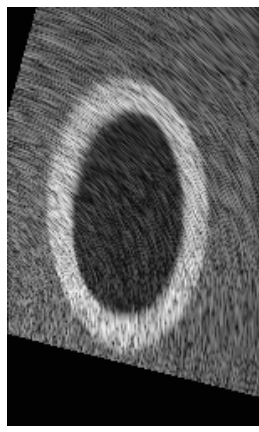

(e)

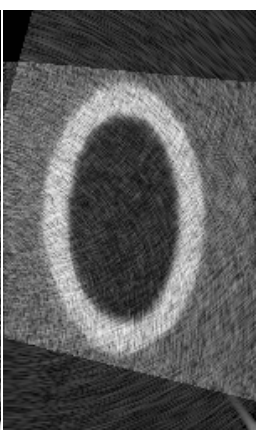

(b)

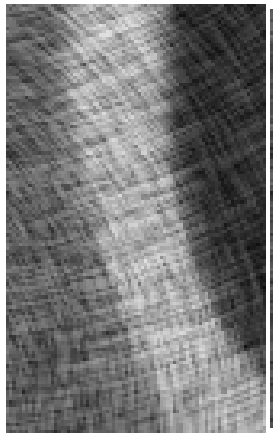

(f)

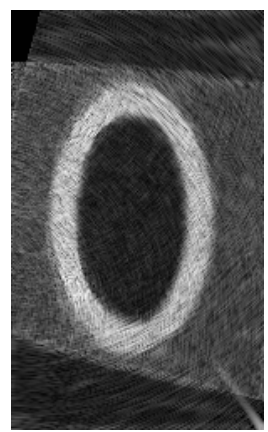

(c)

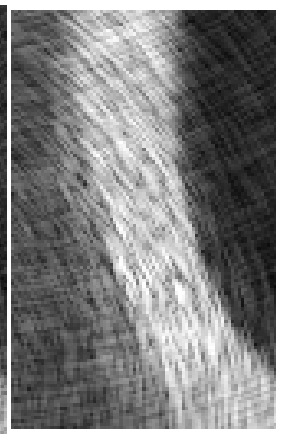

(g)

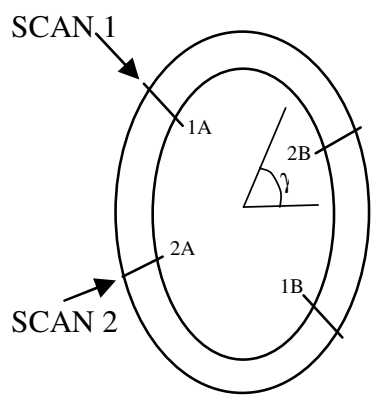

(d)

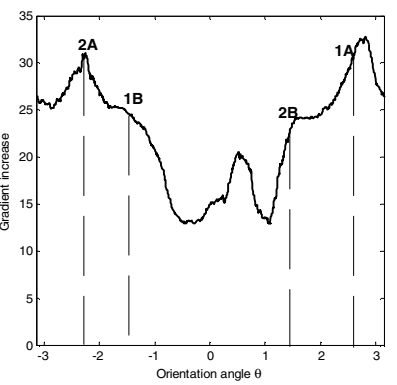

(h)

Fig. 1. Two views of a synthetic phantom (a, e). Comparison of the compounding results using intensity averaging (b) and our algorithm (c). Magnified views of a smaller region are presented in (f), for the intensity averaging, and in (g) for our algorithm. Note the contrast increase and noise reduction. (d): schematic depiction of the location and orientation of the probe, relative to the scanned ring, in both scans. Points where the ultrasound beam is approximately normal to the ring are marked $(1 \mathrm{~A}, 1 \mathrm{~B}, 2 \mathrm{~A}, 2 \mathrm{~B})$. (h): Increase in gradient magnitude at the ring contour with our method, when compared to intensity averaging. The horizontal coordinate represents the orientation angle $\gamma$. As expected, the maximum increase is obtained where the beam is approximately perpendicular to the ring.

integrated way by using the monogenic signal. The use of phase provides the benefit of contrast invariance, which can be key in ultrasound image processing.

An additional advantage of the presented technique is that the framework we propose is independent on the actual selection of the functions that quantify structural information and orientation. For other applications, it would be possible to introduce alternatives, while keeping the main ideas from our work. Particularly, in some cases the successful use of phase congruency for the estimation of feature significancy can be quite dependent on the selected scales and the choice of function $g\left(S_{i}\right)$. Other techniques might prove to be more robust than phase congruency for this purpose.

The introduction of a constant $\alpha$ in (2) allows to control the amount of speckle reduction depending on the application. For visual diagnosis, it can be dangerous to remove information from the original image, as important information could be there; 


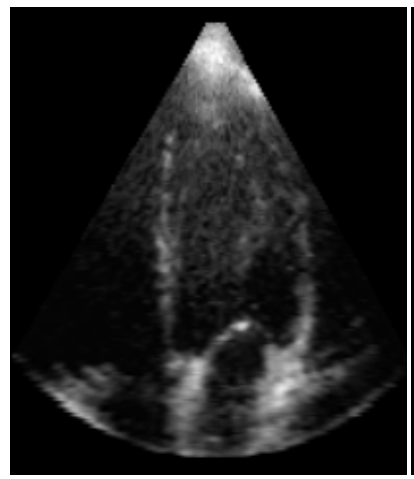

(a)

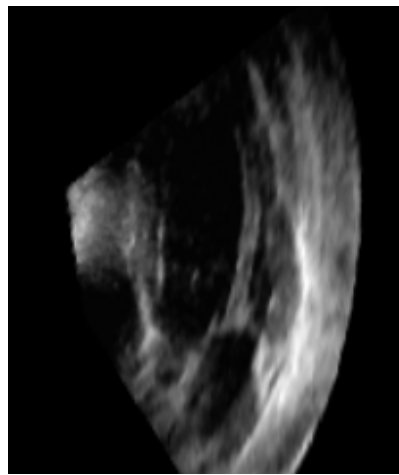

(d)

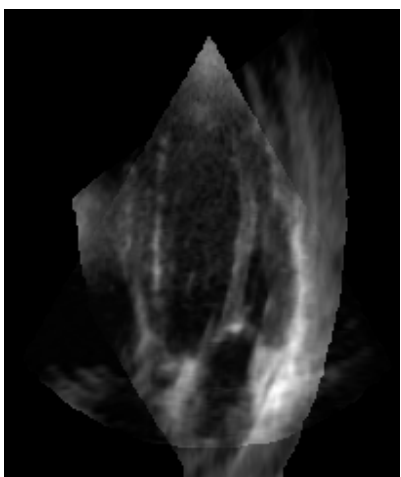

(b)

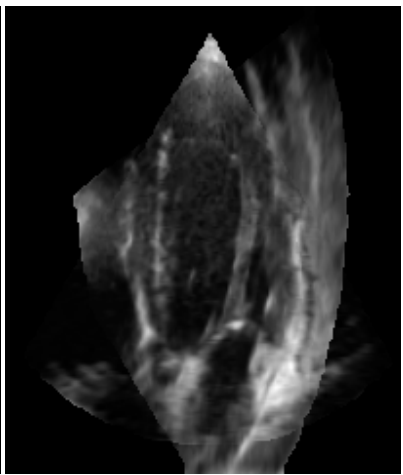

(e)

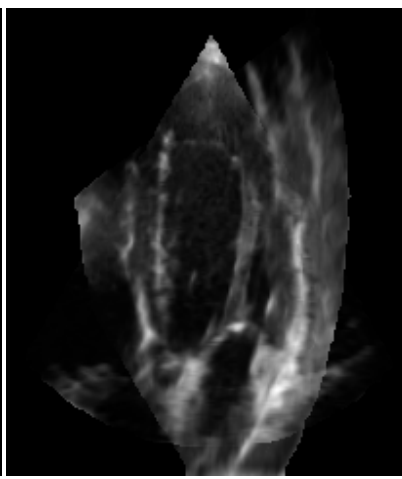

(c)

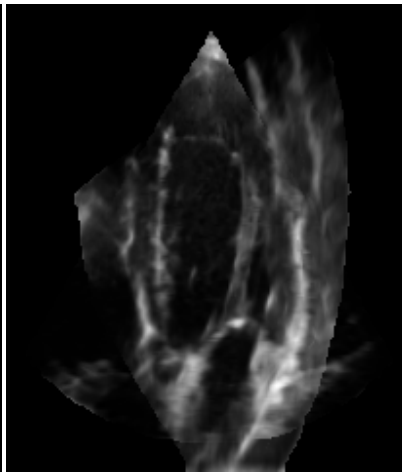

(f)

Fig. 2. Application of our method to RT3DUS images. (a): original apical image. (d): Original parasternal image. (b): Intensity averaging. (e): Our method with $\alpha=1$. Note the enhancement of the vertical structure on the right and left sides of the image. (c): Our method with $\alpha=0.9$ : apart from the structure enhancement, a significant noise reduction is obtained. (f): Our method with $\alpha=0.6$ : a strong noise reduction is applied without loss of structure definition. Differences are better appreciated in the animations provided online as additional material.

while for automatic segmentation algorithms, a drastic reduction of the speckle content can be advisable. Another possibility would be to keep the speckle removed from one image (the last term in (2)) and display it separately: significant information about aspects such as motion could be obtained from it. Planned clinical studies will provide us with more insight on this.

The band-pass filters used in the method have to be selected, depending on the size of the structures we want to detect. For applications in which the basic structures are always similar (e.g., echocardiography), filter frequencies can be kept unmodified for all patients.

Though registration has not been addressed in the present work, it is a fundamental task and the results will depend dramatically on the registration accuracy. Initially, as the images are acquired from the same patient with a few minutes between them, we expect rigid registration to be accurate enough for our purposes; however, patient 
movement or breathing may prove to produce heart deformation that would make an elastic registration step necessary.

Preliminary results on simulated and real ultrasound images show a significant improvement, both in visual quality and quantitative measurements. Further validation, including segmentation algorithms applied on the original and combined images, is currently under way in our group.

\section{Acknowledgments}

The authors wish to thank Drs. Harald Becher and Carla Lofiego, from the John Radcliffe Hospital in Oxford, for providing the datasets used in this study. Funds were provided by the EPSRC, grant GR/S72801.

\section{References}

1. Leotta, D.F., Martin, R.W., "Three-dimensional spatial compounding of ultrasound scans with weighting by incidence angle", Ultrasonic Imaging 22, 1-19 (2000)

2. Felsberg, Sommer, "The monogenic signal", IEEE Transactions on Signal Processing, 49(12):3136-3144, (2001)

3. Boukerroui, D., Noble, J.A., Brady, M., "On the choice of band-pass quadrature filters", Journal of Mathematical Imaging and Vision, 21:53-80 (2004)

4. Morrone, M.C., Owens, R.A., "Feature detection from local energy", Pattern Recognition Letters, 6, 303-313 (1987)

5. Kovesi, P., "Phase congruency: a low level image invariant", Psych Res 64: 136-148 (2000)

6. Mulet-Parada, M., Noble, J.A., "2D+T Acoustic Boundary Detection in Echocardiography”, Medical Image Analysis, 4(1):21-30 (2000)

7. Jensen, J.A. "Field: A Program for Simulating Ultrasound Systems", Medical \& Biological Engineering \& Computing 34: 351-353 (1996) 